\title{
Benefits of extra-pair mating may depend on environmental conditions - an experimental study in the blue tit (Cyanistes caeruleus)
}

\author{
Aneta Arct • Szymon M. Drobniak • Edyta Podmokła • \\ Lars Gustafson • Mariusz Cichoń
}

Received: 17 March 2013 / Revised: 26 June 2013 / Accepted: 27 June 2013 / Published online: 17 July 2013

(C) The Author(s) 2013. This article is published with open access at Springerlink.com

\begin{abstract}
Extra-pair mating constitutes a relatively common reproductive strategy in many socially monogamous bird species. This strategy may considerably improve reproductive success of males, but female benefits from extra-pair matings still remain unclear and empirical evidence is scarce. This may be because genetic benefits of extra-pair mating are not always revealed. It is possible that they are shown only in unfavourable environmental conditions and hence problems arise with detecting differences between within- and extrapair offspring whose performance is measured under favourable conditions. In order to test this prediction, we manipulated environmental conditions by altering brood sizes of blue tits and compared phenotypic characteristics of within- and extra-pair offspring in mixed-paternity broods. We found that extra-pair young exhibited a higher response to phytohemagglutinin in comparison to within-pair young, but this was only observed among nestlings from experimentally enlarged broods. These results indicate that genetic benefits may interact with the environment, and thus benefits of extrapair mating are likely to become visible only when conditions are relatively unfavourable.
\end{abstract}

Keywords Extra-pair paternity $\cdot$ Birds $\cdot$ Cell-mediated immunity $\cdot$ Indirect benefits

Communicated by C. M. Garcia

A. Arct $(\bowtie) \cdot S$. M. Drobniak $\cdot$ E. Podmokła $\cdot$ M. Cichoń Institute of Environmental Sciences, Jagiellonian University, Gronostajowa 7, 30-387 Kraków, Poland

e-mail: aneta.arct@uj.edu.pl

\section{Gustafson}

Department of Ecology and Genetics/Animal Ecology, Evolutionary Biology Centre (EBC), Uppsala University, Uppsala, Sweden

\section{Introduction}

Extra-pair mating constitutes a relatively frequent reproductive strategy in a number of passerine species (Petrie and Kempenaers 1998). Although males may clearly benefit from this behaviour, which enables them to sire more offspring, female benefits from extra-pair copulation (EPC) are still debated (Griffith et al. 2002; Akçay and Roughgarden 2007; Griffith and Immler 2009). In socially monogamous species, direct benefits of extra-pair copulation are assumed to be rare (Griffith et al. 2002). Thus, indirect benefits in terms of genetic profits to the offspring are usually invoked to explain this intriguing female behaviour (Jennions and Petrie 2000; Neff and Pitcher 2005, but see Forstmeier et al. 2011). Such benefits may stem from additive genetic effects or interactions between maternal and paternal genes (non-additive effects; e.g. Foerster et al. 2003). Both scenarios predict that young resulting from EPCs should on average be of superior quality (Griffith et al. 2002; Akçay and Roughgarden 2007). Indeed, some studies showed improved quality of extra-pair offspring in a number of fitness-related traits (Kempenaers et al. 1997; Sheldon et al. 1997; Johnsen et al. 2000). However, many other studies failed to show any differences between within- and extra-pair offspring (reviewed by Akçay and Roughgarden 2007). This inconsistency of results can be accounted for by the fact that genetic effects, although biologically significant, might be subtle. Thus, relatively large sample sizes may be needed to detect them (Schmoll 2011). Moreover, the genetic benefits of extra-pair matings may become meaningful only under particular environmental conditions (Schmoll 2011). They may be revealed in competitive environments but appear nonsignificant under favourable conditions.

Life history traits, in particular immunity, are often moulded by environment-specific gene expression (e.g. Stearns 1992). 
Thus, if genetic benefits of extra-pair matings are differently expressed across environments, such genetic-environmental interaction may make it difficult to detect potential benefits from extra-pair matings (Schmoll 2011). These benefits may be detectable in some ecological circumstances, but may appear too small or non-existent in others. For instance, in the study on the Common Yellowthroat (Geothlypis trichas) extrapair offspring was shown to have a higher cellular immune response but this was only detected in the colder breeding season (Garvin et al. 2006). Schmoll et al. (2005) showed that extra-pair offspring (EPO) had a higher probability of local recruitment into the breeding population, but only when it originated from late broods, experiencing poorer environmental conditions.

The studies of Garvin et al. (2006) and Schmoll et al. (2005) clearly suggest that genetic benefits may indeed interact with environmental conditions. However, such a possibility has not been directly tested in experimental studies. Here, we present results of a brood size manipulation experiment, performed in a nest box population of blue tits, in which we compared the performance of within- and extrapair nestlings. We expected nestlings originating from extrapair matings to perform better in comparison to their half siblings originating from within-pair matings and we expected the superior quality of extra-pair offspring to be better expressed among experimentally enlarged broods. Hence, we searched for a significant interaction between nestling paternity and environmental conditions. Observation of such genetic-environmental interaction would significantly contribute to our understanding of the nature of indirect genetic benefits from extra-pair matings and explain why many studies have failed to detect such benefits.

\section{Material and methods}

\section{Study species}

The study was conducted during the breeding seasons of 2009 , 2010 and 2011 in the population of blue tits breeding in nest boxes on a Swedish island, Gotland $\left(57^{\circ} 03^{\prime} \mathrm{N}, 18^{\circ} 17^{\prime} \mathrm{E}\right.$; see Pärt and Gustafsson (1989) for more detailed description of the study area). The blue tit (Cyanistes caeruleus) is a small migrant passerine from the Paridae family. In the Gotland population, the female blue tits lay one clutch per season, consisting on average of 10-12 eggs and incubate the clutch alone for approximately 2 weeks. Nestlings are fed by both parents and the young fledge after 16-22 days. After fledging, the young are fed by the parents for another 2 weeks. The blue tit is a model species particularly suitable to study mating strategies. In the Gotland population, the blue tits is a monogamous species but extra-pair copulation is common and occurs in over $40 \%$ of broods. Extra-pair offspring have a larger body size (Dreiss et al. 2008) and are more likely to fledge than their within-pair half siblings (Kempenaers et al. 1997; Charmantier et al. 2004).

Field techniques

From the end of April, we regularly inspected nest boxes to determine the laying date, clutch size and hatching success. To create standard differences in rearing conditions, we manipulated brood size. We selected pairs of broods with the same hatching date and a similar brood size ( \pm 1 chick). One randomly selected brood in each pair was enlarged (experimental nest) by adding three nestlings from a donor nest. These extra nestlings from donor nest served only to increase within-nest competition and were not used in the analyses. This equals ca. $30 \%$ increase in brood size. Brood enlargement appears to be an effective way of increasing competition within the brood. Negative effects of brood size enlargement on various nestling traits have been reported (e.g. Cichoń and Dubiec 2005). In addition to brood size manipulation, half of randomly chosen nestlings were crossfostered between the control and the enlarged nest within each pair. Thus, nestlings originating from one family were raised under different environmental condition. Brood size manipulation and cross-fostering were performed on the second day after hatching. All nestlings were individually marked by clipping their nails, blood-sampled and weighed with an electronic balance (to the nearest $0.1 \mathrm{~g}$ ). Nestlings were weighted again on days 11, 12 and 14. Tarsus length was measured at day 14 with an electronic calliper to the nearest $0.1 \mathrm{~mm}$ and the birds were then marked with aluminium rings.

We measured offspring performance in terms of their body mass, tarsus length and cell-mediated immune response to a nonpathogenic antigen. Immunocompetence appears to provide an adequate framework to study our question because it has frequently been shown to be a heritable and condition-dependent trait (Saino et al. 1997; Brinkhof et al. 1999; Cichoń et al. 2006; Drobniak et al. 2010) and to predict survival (Christe et al. 1998; Cichoń and Dubiec 2005) and longevity (Birkhead et al. 1999). Hence, genes responsible for enhanced immune function may constitute an important target of female extra-pair mate choice.

Adults were caught inside nest boxes or by mist nets while feeding 14-day-old nestlings. They were weighed, measured and marked with an aluminium ring. Adult sex was determined by the presence of a brood patch. Blood samples (ca. $20 \mu \mathrm{l}$ ) were collected from all adults and stored in $96 \%$ ethanol for further genetic analyses.

Assessing the immune function

To assess nestling immunocompetence, on day 11 after hatching, nestlings were injected with a non-pathogenic antigen, phytohemagglutinin (PHA; Sigma). This method is widely used for 
assessment of immunocompetence in birds (Lochmiller et al. 1993). Injection with PHA results in local activation and proliferation of T-cells followed by local recruitment of inflammatory cells and increased expression of major histocompatibility complex molecules (Goto et al. 1978). Briefly, the solution of $0.2 \mathrm{mg}$ PHA in $0.04 \mathrm{ml}$ saline was injected subcutaneously into the wing web. Before the injection and $24 \mathrm{~h}$ later, the thickness of the wing web was measured with a pressure-sensitive calliper by the same person (see Drobniak et al. (2010) for details). The difference between wing thickness prior to and $24 \mathrm{~h}$ after the injection was a measure of the immune response to the antigen.

\section{Molecular paternity analyses}

DNA was extracted with Chelex according to a standard protocol. Nestling sex was determined using P2 and P8 primers (Griffiths et al. 1998). The number of microsatellite loci used to determine parentage varied between years. In 2009 and 2010, the paternity was identified using five microsatellite loci: Pca3, Pca4, Pca8, PmaTGAn45 and Titgata79. In 2011, we used an additional microsatellite locus Pca7. PCRs for the Pca3, Pca4 and Pca8 markers were performed as described in Dawson et al. (2000) and for PCA7, PmaTGAn45 and Titgata79 as described in OlanoMarine et al. (2010). The annealing temperature in multiplex reactions was set to $53{ }^{\circ} \mathrm{C}$. PCR products, obtained with fluorescently labelled primers, were analysed on ABI 3031 sequencer and using GeneMaper software. Marker polymorphism and exclusion probabilities based on genetic data of all individuals sampled $(N=673)$ were calculated in CERVUS v3.0 (Kalinowski et al. 2007). The five microsatellite markers constituted a powerful marker set for parentage analyses in blue tits (polymorphism in total number of alleles: Pca3, 38; Pca8, 64; Pca4, 27; PmaTGAn45, 43; Titgata79, 74; Pca7, 15), with a combined exclusion probability of the first parent greater than 0.999 and for the second parent 0.999 . We assumed that mismatches between social paternal and offspring genotypes at a single locus were mutations, and mismatches at two or more loci resulted from extra-pair paternity. Overall, 55 out of 673 offspring were sired by extra-pair males in 30 out of 72 successfully analysed broods. Five out of 55 extra-pair young did not survive until day 14.

\section{Statistics}

To test for differences in the immune response, body mass and tarsus length between extra-pair and within-pair nestlings, a linear mixed model (Proc MIXED in SAS with Restricted Maximum Likelihood method) was applied. We used only broods in which mixed paternity was detected. The data structure for testing differences between extra-pair and within-pair young was highly unbalanced due to a much larger number of nestlings coming from within-pair matings in comparison to nestlings from extra-pair matings, but the employed technique is appropriate to deal with unbalanced data (Shaw 1987).

In our experimental design, within- and extra-pair nestling originating from one family were supposed to be raised under different environmental conditions. Consequently, it may allow disentangling the genetic and environmental sources of variation. Unfortunately, due to the relatively small sample size formal statistical analyses concerning variance components related to the nest of origin, nest of rearing and their interaction with paternity are not fully supported. The identity of the brood of origin and the brood of rearing was included as random factors to account for the cross-fostering procedure; hence, we controlled for the fact that some chicks (both WPO and EPO) remained in their parental nests but some were raised in foster nests. In total, there were 26 nests of origin. However, as some chicks from mixed-paternity broods were reared in nests without any extra-pair young, the number of nest-of-rearing was greater and equals 43 . Experimental treatment, paternity (two levels, within and extra-pair), offspring sex and year of study were defined as fixed factors. Controlling for the sex effect is important because blue tit nestlings show sexual dimorphism in the traits considered here. In the analysis of the T-cell-mediated immune response, we used the body mass at day 12 (when the immune response was measured) as a covariate. The interaction between experimental treatment and paternity was the effect of our main interest and thus remained in the model even if non-significant. The remaining interactions were tested but discarded if non-significant to increase the power of the test, which is particularly important given the relatively small sample size. Post hoc analyses (linear mixed model) were performed within groups in the case of significant interactions. These models included the random factors as in the original model and appropriate fixed factors excluding the one being the subject of within group analyses. Sample sizes differ between the analyses as some measurements were not always available for all nestlings.

\section{Results}

The proportion of extra-pair young in mixed paternity broods did not differ between years $\left(\chi_{2}{ }^{2}=0.88, p=0.644\right)$, sexes $\left(\chi_{1}{ }^{2}=0.87, p=0.768\right)$ and experimental groups $\left(\chi_{1}{ }^{2}=1.97\right.$, $p=0.161)$. We found a significant interaction between experimental treatment and paternity in the analyses of the cell-mediated immune response (Table 1; Fig. 1). The comparisons performed within experimental groups revealed that extra-pair young had greater immune responses than within-pair young among enlarged broods $\left(F_{1,108}=5.42\right.$, $p=0.022$ ), while no difference between extra-pair and within- 
Table 1 Mixed model analyses of the immune response to PHA, tarsus length and body mass of nestlings originating from extrapair and within-pair matings.

Nest of origin and nest of rearing were included as higher level random effects (results not shown), while paternity, experimental treatment, sex, body mass and year were defined as fixed factors

\begin{tabular}{llcc}
\hline Source of variation & $d f$ & $F$ & $p$ \\
\hline T-cell-mediated immune response & & & \\
Year & $2,24.5$ & 2.92 & 0.073 \\
Sex & 1,218 & 0.11 & 0.738 \\
Paternity & 1,211 & 1.10 & 0.296 \\
Experiment & $1,44.9$ & 1.88 & 0.177 \\
Paternity $\times$ experiment & 1,216 & 4.83 & 0.030 \\
Mass (day 12) & 1,166 & 23.93 & $<0.0001$ \\
Tarsus length on day 14 & & & \\
Year & 2,24 & 2.35 & 0.117 \\
Sex & 1,221 & 38.93 & $<0.0001$ \\
Paternity & 1,219 & 0.25 & 0.615 \\
Experiment & $1,38.7$ & 2.32 & 0.136 \\
Paternity $\times$ experiment & 1,219 & 1.41 & 0.237 \\
Body mass on day 14 & & & \\
Year & 2,30 & 1.95 & 0.160 \\
Sex & 1,218 & 33.11 & $<0.0001$ \\
Paternity & 1,215 & 1.63 & 0.204 \\
Experiment & 1,39 & 0.29 & 0.594 \\
Paternity $\times$ year & 2,216 & 4.06 & 0.018 \\
Paternity $\times$ experiment & 1,216 & 0.63 & 0.430 \\
\hline
\end{tabular}

pair young was found among control broods $\left(F_{1,106}=1.13\right.$, $p=0.290$; Fig. 1). Body mass on day 14 was affected by an interaction between paternity and year. To explore this relationship further, we performed additional analyses separately for each year. Extra-pair young had a lower body mass than within-pair young in 2011 while non-significant differences were observed in 2009 and 2010 (Fig. 2). Extrapair young did not differ from within-pair young in terms of tarsus length on day 14. More importantly, the interaction between experimental treatment and paternity appeared nonsignificant for body mass and tarsus length (Table 1).

\section{Discussion}

In our study, $41.7 \%$ of broods contained at least one extra-pair offspring. The observed frequency of extra-pair paternity lies within a range recorded in other populations of this species (Kempenaers et al. 1997; Charmantier et al. 2004; Krokene et al. 1998; Leech et al. 2001; Brommer et al. 2007; Magrath et al. 2009). As female blue tits actively seek extra-pair mating (Kempenaers et al. 1992), such behaviour should be associated with some benefits to the female. In line with this prediction, we found that extra-pair offspring showed a higher immune response to PHA. The superior quality of extra-pair offspring has already been shown in some previous studies on passerine birds, including blue tits (e.g. Kempenaers et al. 1997; Foerster et al. 2003; Dreiss et al. 2008), but a number of studies failed to find the expected differences between maternal half-sibs (e.g. Whittingham et al. 2003; Schmoll et al. 2003; Kleven et al. 2006; reviewed in Akçay and Roughgarden 2007). Schmoll (2011) suggests that such inconsistency between studies may result from the fact that the genetic fitness benefits from extrapair mating may be expressed only in some environments while remain undetected in others. Our results clearly confirm such a possibility. We experimentally manipulated rearing conditions and showed that potential benefits from extra-pair matings may be revealed only in a more competitive environment. Specifically, we found that EPO showed a stronger immune response than WPO among enlarged broods, while no differences were observed among control broods (Fig. 1). The environment-depended trait expression of EPO young is further confirmed by the significant interaction between paternity and year, observed in the analyses of body mass on day 14. In this case, EPO appeared to show a lower body mass than WPO in one of three study years. This is somewhat counter intuitive but shows that expression of extrapair genes might be complex and dependent on the environment. It may also show that extra-pair mating may not always be as advantageous as commonly assumed. Genotype by environment interactions have been repeatedly reported in numerous population genetics studies. However, such interactions seem to have been neglected so far in studies of alternative mating strategies. The paternity by year interaction 
Fig. 1 The immune response to PHA of within-pair offspring (WPO solid line) and extra-pair offspring (EPO dashed line) from control and experimental nests (enlarged broods). Results of post hoc analyses (linear mixed model, see "Material and methods" section for details on statistics). Least squared means with standard errors are presented. Line with asterisk connects significantly different groups $(p<0.05)$

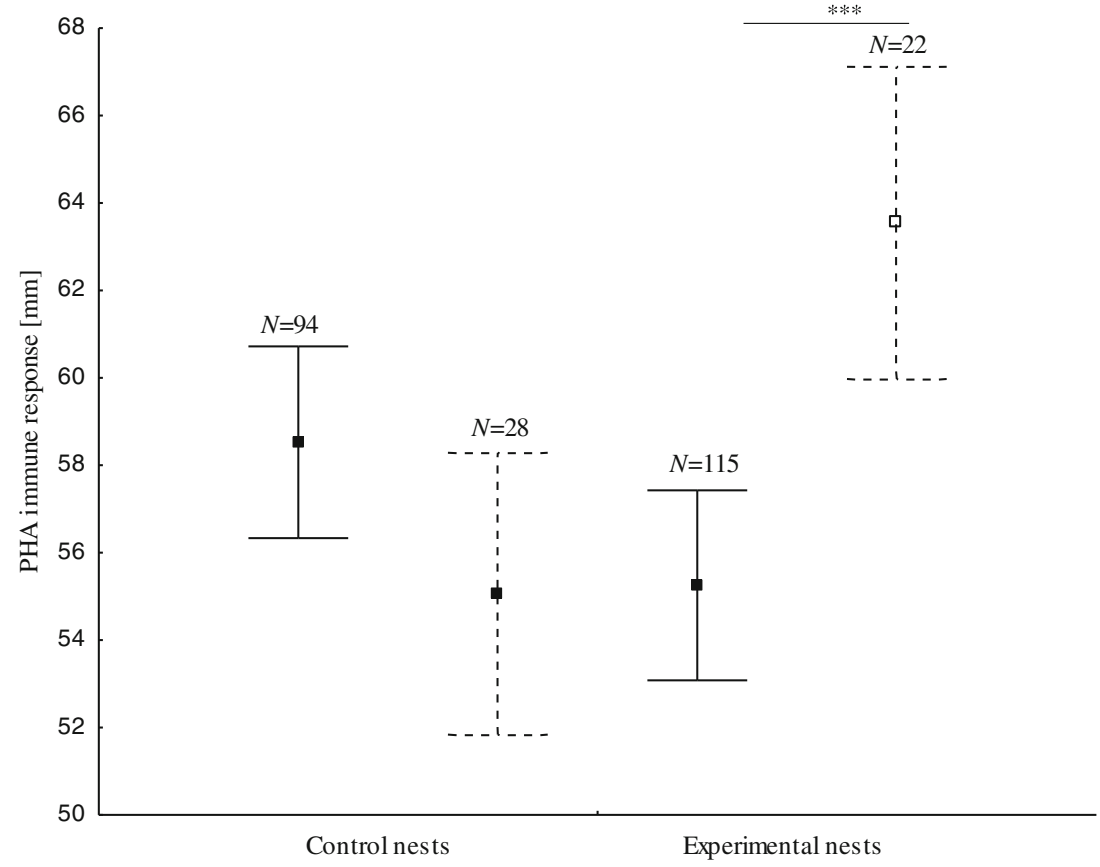

was not significant in the analyses of the PHA response (effect removed from the final model). EPO and WPO did not differ in tarsus length (Table 1). Hence, genes of extra-pair males appeared to enhance offspring immunocompetence without any corresponding effects on tarsus length.

In our study, the potential superior quality of extra-pair nestlings was expressed only in the immune response to PHA. The importance of immune function in determining fitness components seems to be very intuitive, as it translates directly to survival prospects in environments hosting an array of pathogens. Thus, a higher immune response of extra- pair offspring in comparison to within-pair offspring may indeed generate significant fitness benefits. Possibly, extrapair offspring inherit specific immunity genes and female choice of an extra-pair mate might be focused on obtaining such genes. However, the observed differences in performance between extra-pair and within-pair offspring may potentially be explained by maternal effects (e.g. Magrath et al. 2009; Tschirren et al. 2011). Unfortunately, our experimental design does not allow separating genetic and early maternal effects. Thus, the observed differences may potentially result from differential female allocation of
Fig. 2 The body mass of withinpair offspring (WPO solid line) and extra-pair offspring (EPO dashed line) in the 3 years of study. Results of post hoc analyses (linear mixed model, see "Material and methods" section for details on statistics). Least squared means with standard errors are shown. Line with asterisk connects significantly different groups $(p<0.05)$

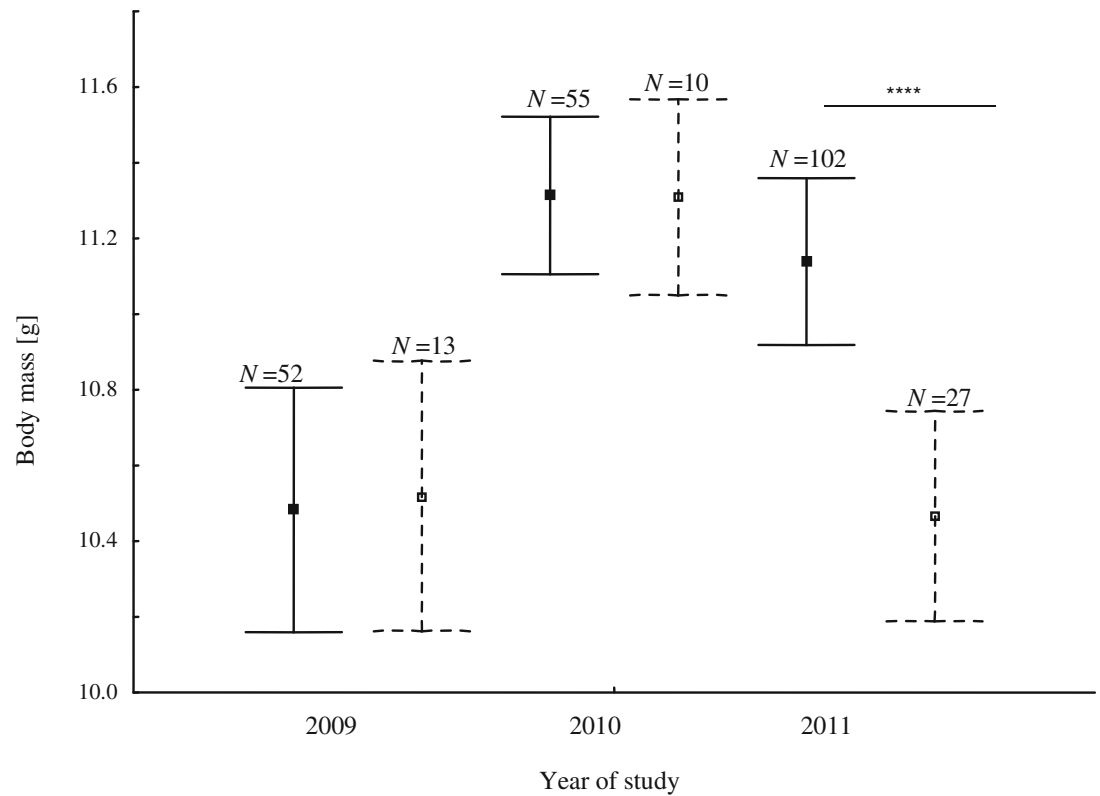


specific maternal components into the eggs (Tschirren et al. 2011) or from non-random distribution of EPO in the laying order (Magrath et al. 2009; Ferree et al. 2010). Immune function is known to improve with nestling age, and early hatched chicks exhibit greater immunity than their younger siblings (Roulin et al. 2007). In fact, both genetic and maternal effects might interact to result in observed differences between extra-pair and within-pair offspring. Thus, the expression of the paternal genes might be very complicated as the genes inherited from extra-pair male might interact with the environmental conditions and maternal effects. Existence of such interactions may be responsible for numerous failures of detecting superior quality of extra-pair offspring.

The development of the immune system and immunity per se is strongly affected by environmental conditions (e.g. Lochmiller and Deerenberg 2000), so only high-quality individuals might be able to mount strong immune response under poor rearing conditions. Thus, one may assume that the observed differences between maternal half siblings may result from higher competitive abilities of extra-pair young expressed in a poor rearing environment. No differences in performance were observed among unaltered control nests, experiencing relatively favourable rearing conditions. Thus, any potential benefits from extra-pair mating may be small or not existing, and so remain undetected in favourable environments (Schmoll 2011). Our result is consistent with some previous studies on passerine bird species showing that genetic benefits from extrapair matings may be revealed only under relatively poor environmental conditions (Schmoll et al. 2003; Garvin et al. 2006; Edler and Friedl 2008). However, in contrast to these previous studies, in our study we experimentally showed that the magnitude of genetic benefits from extra-pair mating depends on the environmental context. Thus, difficulties with demonstrating the expected superior quality of extra-pair young experienced by many previous studies may stem from the fact that in these studies, within and extra-pair nestlings possibly experienced favourable environmental conditions.

Such context-dependent genetic effects presuppose temporal and/or spatial variation in mating strategies (Schmoll 2011). If relevant environmental gradients affecting offspring performance are predictable, one may expect females to show phenotypic plasticity in mating strategies (discussed in Qvarnström et al. 2000). For example, in the coal tit (Periparus ater) the frequency of extra-pair paternity was substantially higher among the second broods (experiencing relatively stressful environmental conditions) compared to the first broods (raised in relatively good environmental conditions; Dietrich et al. 2004). Thus, the context-dependent genetic effects suggest that selection on female mating preferences may vary across environments. This may have interesting implications for the evolution of mating preferences and the maintenance of genetic variation in sexually selected traits. Future research should concentrate on studying such context-dependent genetic benefits and employ experimental manipulations and quantitative genetic techniques to give conclusive evidence.

Acknowledgments We thank Blandine Doligez for providing information on some blue tit nests locations, Justyna Kubacka for correcting English and two anonymous reviewers for their helpful comments. This research was funded by Polish Ministry of Science and Higher Education (to MC) and partly by DS/WBiNoZ/INoŚ/757/09-11.

Ethical standards This study conforms to the legal requirements of Sweden.

Conflict of interest The authors declare that they have no conflict of interest.

Open Access This article is distributed under the terms of the Creative Commons Attribution License which permits any use, distribution, and reproduction in any medium, provided the original author(s) and the source are credited.

\section{References}

Akçay E, Roughgarden J (2007) Extra-pair paternity in birds: review of the genetic benefits. Evol Ecol Res 9:855-868

Birkhead TR, Fletcher F, Pellatt EJ (1999) Nestling diet, secondary sexual traits and fitness in the zebra finch. Proc R Soc Lond B 266:385-390

Brinkhof MWG, Heeb P, KoÈlliker M, Richner H (1999) Immunocompetence of nestling great tits in relation to rearing environment and parentage. Proc R Soc Lond B 266:2315-2322

Brommer JE, Korsten P, Bouwman KM, Berg ML, Komdeur J (2007) Is extrapair mating random? On the probability distribution of extrapair young in avian broods. Behav Ecol 18:895-904

Charmantier A, Blondel J, Perret P, Lambrechts MM (2004) Do extrapair paternities provide genetic benefits for female blue tits Parus caeruleus? J Avian Biol 35:524-532

Christe P, Møller AP, de Lope F (1998) Immunocompetence and nestling survival in the house martin: the tasty chick hypothesis. Oikos 83:175-179

Cichoń M, Dubiec A (2005) Cell-mediated immunity predicts the probability of local recruitment in nestling blue tits. J Evol Biol 18:962-966

Cichoń M, Sendecka J, Gustafsson L (2006) Genetic and environmental variation in immune response of collared flycatcher nestlings. $\mathrm{J}$ Evol Biol 19:1701-1706

Dawson DA, Hanotte O, Greig C, Stewart IRK, Burke T (2000) Polymorphic microsatellites in the blue tit Parus caeruleus and their cross-species utility in 20 songbird families. Mol Ecol 9:1941-1944

Dietrich V, Schmoll T, Winkel W, Epplen JT, Lubjuhn T (2004) Pair identity: an important factor concerning variation in extra-pair paternity in the coal tit (Parus ater). Behaviour 141:817-835

Dreiss AN, Silva N, Richard M, Moyen F, Thery M, Møller AP, Danchin E (2008) Condition-dependent genetic benefits of extrapair fertilization in female blue tits Cyanistes caeruleus. J Evol Biol $21: 1814-1822$

Drobniak SM, Wiejaczka D, Arct A, Dubiec A, Gustafsson L, Cichoń M (2010) Sex-specific heritability of cell-mediated immune response in the blue tit nestlings (Cyanistes caeruleus). J Evol Biol 23:1286-1292

Edler R, Friedl TWP (2008) Within-pair young are more immunocompetent than extrapair young in mixed-paternity broods of the red bishop. Anim Behav 75:391-401 
Ferree ED, Dickinson J, Rendell W, Stern C, Porter S (2010) Hatching order explains an extrapair chick advantage in western bluebirds. Behav Ecol 21:802-807

Foerster K, Delhey K, Johnsen A, Lifjeld JT, Kempenaers B (2003) Females increase offspring heterozygosity and fitness through extra-pair matings. Nature 425:714-717

Forstmeier W, Martin K, Bolund E, Schielzeth H, Kempenaers B (2011) Female extra-pair mating behaviour can evolve via indirect selection on males. P Natl Acad Sci USA 108:10608-10613

Garvin JC, Abroe B, Pedersen MC, Dunn PO, Whittingham LA (2006) Immune response of nestling warblers varies with extra-pair paternity and temperature. Mol Ecol 15:3833-3840

Goto N, Kodama H, Okada K, Fujimoto Y (1978) Suppression of phytohemagglutinin skin response in thymectomized chickens. Poultry Sci 57:246-250

Griffith SC, Immler S (2009) Female infidelity and genetic compatibility in birds: the role of the genetically loaded raffle in understanding the function of extra-pair paternity. J Avian Biol 40:97-101

Griffith SC, Owens IPF, Thuman KA (2002) Extra-pair paternity in birds: a review of interspecific variation and adaptive function. Mol Ecol 11:2195-2212

Griffiths R, Double M, Orr K, Dawson R (1998) A DNA test to sex most birds. Mol Ecol 7:1071-1075

Jennions MD, Petrie M (2000) Why do females mate multiply? A review of the genetic benefits. Biol Rev 75:21-64

Johnsen A, Andersen V, Sunding C, Lifjeld JT (2000) Female bluethroats enhance offspring immunocompetence through extra-pair copulations. Nature 406:296-299

Kalinowski ST, Taper ML, Marshall TC (2007) Revising how the computer program CERVUS accommodates genotyping error increases success in paternity assignment. Mol Ecol 16:1099-1106

Kempenaers B, Verheyen GR, Van den Broeck M, Burke T, Van Broeckhoven C, Dhondt AA (1992) Extra-pair paternity results from female preference for high-quality males in the blue tit. Nature 357:494-496

Kempenaers B, Verheyren GR, Dhondt AA (1997) Extrapair paternity in the blue tit (Parus caeruleus): female choice, male characteristics, and offspring quality. Behav Ecol 8:481-492

Kleven O, Jacobsen F, Izadnegahdar R, Robertson RJ, Lifjeld JT (2006) No evidence of paternal genetic contribution to nestling cellmediated immunity in the North American barn swallow. Anim Behav 71:839-845

Krokene C, Rigstad K, Dale M, Lifjeld JT (1998) The function of extrapair paternity in blue tits and great tits: good genes or fertility insurance? Behav Ecol 9:649-656

Leech DI, Hartley IR, Stewart IRK, Griffith SC, Burke T (2001) No effect of parental quality or extrapair paternity on brood sex ratio in sex ratio in the blue tit Parus caeruleus. Behav Ecol Sociobiol 12:674-680

Lochmiller RL, Deerenberg C (2000) Trade-offs in evolutionary immunology: just what is the cost of immunity? Oikos 88:87-98
Lochmiller R, Vestey M, Boren J (1993) Relationship between protein nutritional status and immunocompetence in northern Bobwhite chicks. Auk 110:503-510

Magrath MJL, Vedder O, van der Velde M, Komdeur J (2009) Maternal effects contribute to the superior performance of extra-pair offspring. Curr Biol 19:792-797

Neff BD, Pitcher TE (2005) Genetic quality and sexual selection: an integrated framework for good genes and compatible genes. Mol Ecol 14:19-38

Olano-Marin J, Dawson DA, Girg A, Hansson B, Ljungqvist M, Kempenaers B, Mueller JC (2010) A genome-wide set of 106 microsatellite markers for the blue tit (Cyanistes caeruleus). Mol Ecol Res 10:516-532

Pärt Y, Gustafsson L (1989) Breeding dispersal in the collared flycatcher (Ficedula albicollis): possible causes and reproductive consequences. J Anim Ecol 58:305-320

Petrie M, Kempenaers B (1998) Extra-pair paternity in birds: explaining variation between species and populations. Trends Ecol Evol 13:52-58

Qvarnström A, Pärt T, Sheldon BC (2000) Adaptive plasticity in mate preference linked to differences in reproductive effort. Nature 405:344-347

Roulin A, Christe P, Dijkstra C, Ducrest A, Jungi TW (2007) Originrelated, environmental, sex, and age determinants of immunocompetence, susceptibility to ectoparasites, and disease symptoms in the barn owl. Biol J Linn Soc Lond 90:703-718

Saino N, Calza S, Möller AP (1997) Immunocompetence of nestling barn swallows in relation to brood size and parental effort. J Anim Ecol 66:827-836

Schmoll T (2011) A review and perspective on context-dependent genetic effects of extra-pair mating in birds. J Ornithol 152:265-277

Schmoll T, Dietrich V, Winkel W, Epplen JT, Lubjuhn T (2003) Longterm fitness consequences of female extra-pair matings in a socially monogamous passerine. Proc R Soc Lond B 270:259-264

Schmoll T, Dietrich V, Winkel W, Epplen JT, Schurr F, Lubjuhn T (2005) Paternal genetic effects on offspring fitness are context dependent within the extra-pair mating system of a socially monogamous passerine. Evolution 59:645-657

Shaw RG (1987) Maximum-likelihood approaches applied to quantitative genetics of natural populations. Evolution 41:812-826

Sheldon BC, Merilä J, Qvarnström A, Gustafsson L, Ellegren H (1997) Paternal genetic contribution to offspring condition predicted by size of male secondary sexual character. Proc R Soc Lond B 264:297-302

Stearns SC (1992) The evolution of life histories. Oxford University Press, New York

Tschirren B, Postma E, Rutstein AN, Griffith SC (2011) When mothers make sons sexy: maternal effects contribute to the increased sexual attractiveness of extra-pair offspring. Proc R Soc Lond B 279:1233-1240

Whittingham LA, Dunn PO, Clotfelter ED (2003) Parental allocation of food to nestling tree swallows: the influence of nestling behaviour, sex and paternity. Anim Behav 65:1203-1210 